\title{
Análisis de las propiedades fisicoquímicas de gasolina y diesel mexicanos reformulados con Etanol
}

\author{
Analysis of Physicochemical Properties of Mexican Gasoline and Diesel \\ Reformulated with Ethanol
}

\author{
Castillo-Hernández P. \\ Departamento de Ingeniería Química \\ Tecnológico de Monterrey, Campus Monterrey \\ Nuevo León, México \\ Correo:pcastillohdz@gmail.com \\ Mendoza-Domínguez A. \\ Departamento de Ingeniería Química \\ Tecnológico de Monterrey, Campus Monterrey \\ Nuevo León, México \\ Correo:mendoza.alberto@itesm.mx
}

\author{
Caballero-Mata P. \\ Centro de Calidad Ambiental \\ Tecnológico de Monterrey, Campus Monterrey \\ Nuevo León, México \\ Correo:pcaballe@itesm.mx
}

Información del artículo: recibido: enero de 2009, reevaluado: junio de 2010, aceptado: mayo de 2011

\section{Resumen}

Los altos precios de los energéticos, la problemática ambiental y las importaciones de combustibles continuamente a la alza, han ocasionado que algunos países redirijan sus esfuerzos al desarrollo de biocombustibles con la finalidad de sustituir parcialmente a los combustibles fósiles. El Etanol es uno de los biocombustibles más usados; Estados Unidos, Brasil y Australia comercializan gasolina con Etanol con una concentración de hasta $85 \%$ en volumen. El presente trabajo muestra los resultados de la caracterización fisicoquímica de mezclas comerciales de gasolina (Magna y Premium) y diesel mexicanos con 10\% y 15\% de Etanol anhidro. Las principales pruebas que se realizaron a los combustibles fueron: Número de Octano de Investigación, Número de Octano del Motor, Número de Cetano, presión de vapor, curva de destilación y poder calorífico. Asimismo, se documentó la estabilidad de las mezclas formuladas. Con los datos de caracterización fisicoquímica obtenidos, se estimaron las emisiones teóricas de $\mathrm{CO}_{2}$ para cada una de las mezclas. Las mezclas de gasolina oxigenadas con Etanol presentaron un aumento en su Número de Octano y un buen Índice de Destilación. El Número de Cetano de las mezclas de diesel disminuyó considerablemente y el poder calorífico, tanto en las mezclas de gasolina como de diesel, se vio afectado negativamente con la adición de Etanol. No obstante, y considerando los créditos por el uso de un combustible renovable, el uso de las mezclas de

\section{Descriptores}

- bioetanol

- biocombustibles

- fuentes móviles

- combustibles reformulados

- gases de efecto invernadero 
gasolina generadas implicaría una disminución teórica máxima de 7.5\% de las emisiones de $\mathrm{CO}_{2}$, mientras que en el caso de las mezclas de diesel representaría un máximo de $9.2 \%$.

\begin{abstract}
High energy prices, environmental issues and increasing importation of fossil fuels has provoked, in some countries, a reorientation of resources towards the development of biofuels that can partially substitute the consumption of fossil fuels. Ethanol is one of the biofuels more commonly used in the world; in the United States, Brazil and Australia gasoline blends that reach up to $85 \%$ Ethanol are commercialized. This work presents the results of a physicochemical characterization of commercial Mexican gasoline (Magna and Premium) and diesel blends with 10\% vol. and 15\% vol. anhydrous Ethanol. The analytical testing included: Research Octane Number, Motor Octane Number, Cetane Number, Reid Vapor Pressure, Distillation Curve and Heating Value. The stability of the blends was also evaluated. The theoretical emissions of $\mathrm{CO}_{2}$ were calculated based on the results of the physicochemical characterization. The ethanol-gasoline blends increased their Octane Number with respect to the commercial gasoline, while conserving an appropriate Distillation Index. The Cetane Number of the ethanol-diesel blends showed a substantial decrease, while the heating value of gasoline and diesel blends was negatively affected by the addition of ethanol. Nevertheless, taking into account the credits by the use of a renewable fuel, the use of the reformulated gasoline blends would imply a maximum theoretical reduction of $7.5 \%$ in $\mathrm{CO}_{2}$ emissions whereas in the case of ethanol-diesel blends it would represent a $9.2 \%$ decrease.
\end{abstract}

\section{Introducción}

El consumo global de energía y las emisiones mundiales de gases de efecto invernadero, principalmente Bióxido de Carbono $\left(\mathrm{CO}_{2}\right)$, han registrado una tendencia ascendente desde 1971 (IPCC, 2001). Los combustibles fósiles son la fuente dominante de energía en el planeta. Su uso intensivo representa el agotamiento de un recurso natural no renovable, además de alterar negativamente la calidad del aire con las emisiones provenientes de su combustión. En México, las emisiones de gases de efecto invernadero por consumo de combustibles fósiles en el 2002, ascendieron a 350,414 Gg, equivalentes a $50 \%$ de las emisiones totales del país, siendo el sector transporte el responsable de $18 \%$ del total de las emisiones nacionales de gases de efecto invernadero (INE, 2006).

La problemática anterior, aunada a los altos precios de los energéticos y a las importaciones de combustibles continuamente al alza, ha traído como consecuencia que parte de la investigación, principalmente en países con reservas de petróleo insuficientes, se dirija hacia el reemplazo total o parcial de los combustibles derivados del petróleo por nuevos combustibles derivados de fuentes renovables. Estos reciben el nombre de biocombustibles, ya que pueden ser producidos a
Keywords:

- bioethanol

- biofuels

- mobile sources

- reformulated fuels

- green-house gases partir de biomasa, incluyendo residuos agrícolas, granos de maíz, paja, legumbres y semillas oleaginosas. Los beneficios ambientales del uso masivo de biocombustibles, como el caso del Etanol, radican en el potencial de reducir significativamente las emisiones netas de $\mathrm{CO}_{2}$, un gas de efecto invernadero, que afectan actualmente al planeta a través del fenómeno de cambio climático que ha sido documentado ampliamente (IPCC, 2007). La reducción en las emisiones de $\mathrm{CO}_{2}$ no es tanto en el proceso de combustión mismo (en donde aún se emite este compuesto) sino que en el proceso global de producción-consumo del biocombustible se devuelve a la atmósfera el $\mathrm{CO}_{2}$ que las plantas absorbieron durante su proceso de crecimiento y desarrollo. Para ser considerado como una alternativa viable, el biocombustible propuesto debe cumplir los siguientes requisitos: proporcionar una ganancia de energía neta, ofrecer beneficios ambientales, ser económicamente competitivo y su producción a gran escala no debe comprometer el abasto de alimentos del país (Hill et al., 2006).

Existe controversia con respecto a la ganancia energética neta del uso de Etanol como sustituto parcial de combustibles fósiles. Algunos autores afirman que es positiva, es decir, que provee más energía durante su consumo que la utilizada durante su producción; sin embargo, otros tantos defienden lo contrario. Esta dis- 
crepancia se debe a que las estimaciones para obtener la ganancia energética neta pueden llegar a ser muy sensibles a las suposiciones hechas con respecto a los límites del sistema usado para los cálculos y a los valores de los parámetros clave. Además, estos cálculos ignoran las diferencias cualitativas entre las diferentes energías fósiles utilizadas en el proceso de obtención, al tomar en cuenta sólo su equivalente térmico, es decir, la energía aportada al proceso (Cleveland, 2005). Con la finalidad de unificar criterios, Farrel et al. (2006) realizaron una comparación de los resultados para la energía neta (energía contenida en el Etanol menos la energía fósil requerida para producirlo) del Etanol publicados por seis diferentes grupos de investigadores, utilizando un modelo que establece los límites del sistema de manera consistente. Los resultados obtenidos indican que con los métodos de producción actuales, el Etanol obtenido a partir de maíz tiene una ganancia neta positiva, podría reducir el uso del petróleo en 95\% con base energética y su uso implicaría una disminución de 13\% de las emisiones de gases de efecto invernadero. A su vez, otros estudios también han demostrado que la ganancia energética del Etanol depende de los insumos utilizados en el proceso y, conforme la tecnología ha avanzado, el proceso de producción de etanol se ha ido optimizando, aumentado así la ganancia energética del Etanol (Gnansounou y Dauriat, 2005).

Sin embargo, el uso de maíz como insumo en la producción de Etanol plantea otro problema, ya que cultivar maíz (como en el caso de Estados Unidos) o caña de azúcar (como en Brasil) en grandes cantidades compromete la seguridad alimentaria e incluso el equilibrio ecológico por la gran cantidad de agua que conlleva tal proceso, el uso de tierras desplaza el cultivo de otros productos e incluso promueve el uso de semillas genéticamente modificadas, que no deja de ser controvertido (Reyes, 2007). En México, el maíz es por mucho el cultivo agrícola más importante: 10.5 millones de toneladas anuales son destinadas al consumo humano. Aunque México es autosuficiente en la producción de maíz (SAGARPA, 2007), es preferible utilizar otros insumos en la producción de Etanol para garantizar su abasto hacia el sector alimenticio y no dar pie a incrementos en el precio del maíz, debidos a especulaciones por parte de intermediarios. Dichos insumos pueden ser: productos maderables, desperdicios agrícolas o materiales celulósicos que no compitan con el consumo humano.

Debido al argumento anterior, se han desarrollado procesos de obtención de Etanol a partir de material celulósico. En el estudio desarrollado por Farrel et al. (2006) se advierte que el Etanol obtenido a partir de ce- lulosa disminuye sustancialmente las emisiones de gases de efecto invernadero y la energía neta es considerablemente mayor que la del Etanol obtenido a partir de maíz. Sin embargo, el costo de producción de Etanol asciende a $\$ 1.50$ dólares por galón si se obtiene a partir de celulosa, mientras que si se usa maíz éste es menos de \$0.90 dólares (McAloon et al., 2000). Por tanto, una de las barreras tecnológicas que impiden el uso del Etanol obtenido a partir de celulosa es precisamente el costo actual de la tecnología.

El uso de Etanol como parte de los combustibles reformulados para vehículos automotor se encuentra ampliamente arraigado en algunos países como Brasil, Estados Unidos y Australia. El primero de ellos comercializa mezclas con un contenido de hasta $85 \%$ en volumen de Etanol en sus gasolinas (E85). En general, todos los automóviles de transporte ligeros a gasolina (conocidos también como los LDV o light-duty vehicles) pueden usar mezclas gasolina-Etanol que contengan menos de $10 \%$ de etanol en volumen (E10), sin necesidad de realizar cambios al vehículo (p. ej., Andersen et al., 2010a; Anderson et al., 2010). En caso de que se requiera usar una mezcla con mayor porcentaje de sustitución de gasolina por Etanol, es necesario hacer modificaciones significativas al vehículo, sobre todo al tren motriz del mismo (Yimin et al., 2007). Existen en el mercado vehículos capaces de utilizar gasolina con $85 \%$ en volumen de Etanol llamados Flex Fuel. Sin embargo, considerando que tan solo en la Zona Metropolitana del Valle de México 29\% de los vehículos en circulación son modelos 1990 y anteriores, resulta poco factible la posibilidad de modificar o cambiar todo el parque vehicular por modelos capaces de utilizar mezclas con un contenido de Etanol mayor por los costos que esto implicaría. Por ello, la reformulación de gasolina con Etanol en un porcentaje bajo es la alternativa que tiene más sentido hoy en día para México.

Así, el objetivo de este estudio es comparar las propiedades fisicoquímicas de la gasolina y el diesel mexicanos, respecto a los correspondientes combustibles reformulados con Etanol a 10\% y 15\% en volumen. Además se evalúan los beneficios ambientales potenciales que conlleva dicha reformulación en cuanto a las emisiones de $\mathrm{CO}_{2}$. La selección de los porcentajes de contenido de Etanol se basó en que las mezclas E10 son de las más comunes (Anderson et al., 2010) y que sustituciones significativamente por encima de este valor requerirían modificaciones al motor de los vehículos que emplearán dichas gasolinas, tal como se comentó, además de que las mezclas con muy bajo contenido de Etanol típicamente presentan poco cambio en sus propiedades respecto a la gasolina base (Hatzioannidis et al., 1998). 


\section{Metodología}

Las mezclas analizadas se prepararon tomando como base de la reformulación gasolinas PEMEX, Magna y Premium comerciales, así como diesel PEMEX también comercial. Lo conveniente hubiera sido realizar a nivel laboratorio la mezcla de gasolinas con el propósito de obtener la contribución directa del Etanol sin la presencia de ningún otro oxigenado. Sin embargo, no fue posible obtener los componentes necesarios para hacerlo así. No obstante, los resultados se estiman valiosos al proveer datos de gasolinas mexicanas reformuladas que no se encuentran comúnmente en la literatura primaria.

Las gasolinas PEMEX Magna, PEMEX Premium y el Diesel PEMEX se oxigenaron con Etanol anhidro grado reactivo a 10 y $15 \%$ en volumen. El principal problema que implica la reformulación de combustibles con Etanol es la estabilidad de la mezcla obtenida, ya que las mezclas de Etanol con hidrocarburos son miscibles sólo con muy pequeñas proporciones de Etanol y su estabilidad se ve influenciada por la temperatura (Gerdes y Suppes, 2001; French y Malone, 2005). En general, temperaturas bajas propician la separación de fases. Por lo tanto, para mantener estables las mezclas a bajas temperaturas típicamente es necesario utilizar aditivos (Karaosmanoğlu et al., 1996; Reyes et al., 2009). Además, el Etanol es altamente higroscópico, por lo que las mezclas de combustible reformuladas con Etanol deben protegerse de la humedad del ambiente (Gramajo de Doz et al., 2004; Mueller et al., 2009).

\section{Estabilidad de las muestras}

Con el fin de preparar mezclas Gasolina-Etanol y Diesel-Etanol estables, a las cuales se les pudieran realizar posteriormente pruebas de sus propiedades fisicoquímicas, se realizaron pruebas previas con un aditivo que fomenta la estabilidad, el cual consistió de una mezcla de alcoholes y acetona: alcohol bencílico ( $20 \%$ vol.), acetona (35\% vol.), alcohol butílico (40\% vol.) y fenol (5\% vol.) (Arnould, 1982). Así mismo, varias investigaciones previas (p. ej. Kwanchareon et al., 2006; Lapuerta et al., 2009) indican que el biodiesel es un aditivo efectivo para la reformulación del Diesel con Etanol, por lo que en el caso del Diesel también se utilizó biodiesel como aditivo para estabilizar las mezclas. El biodiesel que se utilizó se obtuvo de una planta piloto ubicada en las instalaciones del Tecnológico de Monterrey y que emplea aceite comestible gastado como materia prima (Gutiérrez, 2006).

Para determinar la concentración mínima de aditivo necesaria para mantener estables las mezclas a tempe- ratura ambiente se prepararon varias muestras de combustible con aditivo, variando la concentración del mismo entre $1 \%$ y $5 \%$ en volumen. A las mezclas obtenidas se les añadió Etanol gradualmente con una bureta, sin dejar de agitar, hasta completar la cantidad necesaria para obtener mezclas reformuladas con $10 \%$ o $15 \%$ de Etanol en volumen. La aparición de turbidez o una separación de fases era evidencia clara de que se había llegado al límite, en cuanto a solubilidad de Etanol en la mezcla, por lo que en esos casos la mezcla se descartó y se aumentó la cantidad de aditivo hasta obtener la concentración necesaria para mantener estable una mezcla de combustible con Etanol. Como se mencionó anteriormente, la solubilidad es afectada a bajas temperaturas, por lo que también se determinó la estabilidad de las mezclas a temperaturas inferiores a la ambiental. Con base en las pruebas anteriores de estabilidad por mezclado, se prepararon tres muestras con la misma relación Etanol-Combustible (10\% y 15\%) pero diferente cantidad de aditivo (3\%/5\%/10\% y $5 \% / 10 \% / 15 \%$, respectivamente). Cada muestra se preparó por triplicado y acompañada por un blanco muestral (misma composición Etanol-Combustible sin aditivo), para posteriormente almacenarla durante 6 semanas en un baño de temperatura constante. $\mathrm{El}$ rango de temperatura se varió desde $20^{\circ} \mathrm{C}$ hasta $-5^{\circ} \mathrm{C}$, reduciéndose en $5^{\circ} \mathrm{C}$ por semana. Nuevamente la aparición de turbidez fue un indicativo de la inestabilidad de la muestra.

\section{Análisis fisicoquímico de las muestras}

Una vez obtenida la proporción necesaria de aditivo para mantener estables las mezclas, incluso a bajas temperaturas, se procedió a preparar las muestras que se enviarían al laboratorio en el que se realizarían las pruebas fisicoquímicas. Cabe mencionar que las mezclas de gasolina con Etanol se mantuvieron totalmente miscibles, incluso sin la presencia de aditivo y a bajas temperaturas, seguramente por la presencia de algún aditivo previamente adicionado a la mezcla combustible comercial. Por ello, a las mezclas gasolina-etanol que se emplearon para los análisis posteriores no se les agregó aditivo. Las mezclas diesel-etanol si requirieron que se agregara aditivo, tal como se presenta más adelante.

En todos los casos, la preparación de las mezclas se llevó a cabo en un equipo hermético para proteger de la humedad tanto el Etanol anhidro como el combustible oxigenado con Etanol. El mezclador consistió en un recipiente de vidrio de tres bocas conectado a tres bombas peristálticas, las cuales alimentaban el Etanol, el combustible y el aditivo al recipiente. La salida estaba 
conectada a otro recipiente en el cual se recolectaba el combustible reformulado.

Las propiedades fisicoquímicas de las mezclas Gasolina-Etanol que se evaluaron fueron: contenido de oxigenados (ASTM D-5599), Número de Octano de Investigación y Número de Octano del Motor (ASTM D-2699/ ASTM D-2700), Presión de Vapor Reid -PVR- (ASTM D-5191), curva de destilación (ASTM D-86) y estabilidad a la oxidación (ASTM D 525). Para las mezclas Diesel-Etanol, se evaluaron las propiedades fisicoquímicas que se enlistan a continuación: Número de Cetano (ASTM D-613), punto de Inflamabilidad (ASTM D-93) y curva de destilación (ASTM D-86). Se realizaron tres repeticiones por muestra, los análisis mencionados se realizaron en el Southwest Research Institute (San Antonio, Texas). Finalmente, el poder calorífico de los combustibles se determinó mediante el método ASTMD240-02(2007) utilizando un calorímetro marca PARR modelo 6200.

\section{Resultados para las mezclas Gasolina-Etanol}

\section{Contenido de oxigenados}

Como se indicó en la sección anterior, se analizaron tres muestras con diferentes concentraciones de Etanol ( $0 \%, 10 \%$ y $15 \%)$ para cada uno de los tres combustibles fósiles usados (gasolinas Magna y Premium, y Diesel). La cuantificación de contenido de Oxígeno reportado incluye los oxigenados presentes en la gasolina comercial empleada, más la contribución del Etanol. En la tabla 1 se muestra el promedio de los resultados de la prueba de oxigenados para las mezclas de gasolinas Magna y Premium. Se incluyen los valores de Oxígeno total obtenido experimentalmente y las concentraciones individuales de los agentes oxigenantes. Con base en las concentraciones de Etanol obtenidas, se puede confirmar que las muestras fueron preparadas adecuadamente.

El Metil Terbutil Éter (MTBE) es un oxigenado que PEMEX Refinación añade a las mezclas de gasolina Premium y Magna con la finalidad de mejorar el octanaje de las mismas y reducir la concentración de monóxido de carbono en los gases de combustión. El contenido total de Oxígeno aumenta considerablemente en las gasolinas reformuladas con la adición de Etanol, debido a que el contenido de Oxígeno en peso del Etanol es $34.8 \%$, mientras que el del MTBE asciende a $18.2 \%$. Finalmente, el Oxígeno total teórico de las muestras se obtuvo por balance de materia con base en las concentraciones individuales de los oxigenantes y se comparó con los valores experimentales reportados por el laboratorio, obteniéndose una buena coincidencia entre dichos valores (tabla 1).

Número de Octano de Investigación y Número de Octano del Motor

El Número de Octano (NO) de una gasolina es una de sus especificaciones primarias, ya que refleja qué tan propenso es un combustible a causar problemas de "golpeteo" o "cascabeleo" del motor (knocking). La causa de este problema es una autoignición prematura de la mezcla aire-combustible, lo cual afecta el avance del frente de flama dentro del cilindro del motor (Keating, 2007). El Etanol tiene un calor latente de vaporización mayor al de la gasolina, lo cual contribuye a que también tenga un NO relativamente elevado (Anderson et al., 2010). Por tanto, desde hace tiempo se ha reconocido que la adición de Etanol a las gasolinas tiende a mejorar el NO de toda la mezcla, lo cual también se traduce en mejoras en el rendimiento de combustible del motor. 
El NO es el promedio aritmético del valor obtenido por el NOI (Número de Octano de Investigación) y el NOM (Número de Octano del Motor). El NOI es un indicador del desempeño del motor alimentado con la gasolina a evaluar, en condiciones afables. Por otra parte, el NOM proporciona indicios del desempeño del motor en condiciones de operación severas. Por lo general, las gasolinas comerciales tienen un valor de NOI entre 88-101 y un NOM con valores que oscilan entre 80 y 90. El Etanol tiene un NOM reportado de 90 y un NOI de 109 (Anderson et al., 2010), por lo que se utiliza al igual que el MTBE como un potenciador del octanaje como ya se comentó.

Las especificaciones para gasolina PEMEX Magna establecen un valor de NOM de 82 y un número de octano reportado de 87, mientras que para gasolina PEMEX Premium establecen un NOI de 95 y un número de octano reportado de 92 (PEMEX Refinación, 2008). En la tabla 2 se reporta el promedio de los resultados obtenidos de las pruebas realizadas a las diferentes muestras. Tanto el NOI como el NOM aumentaron en el caso de las gasolinas reformuladas con Etanol. En la gasolina Magna, el NOM aumentó $2.9 \%$ con la adición de Etanol a $15 \%$ en volumen, mientras que con gasolina Premium aumentó $1.5 \%$. El NOI en el combustible con Etanol a $15 \%$ en volumen, aumenta $6.5 \%$ en las mezclas con Magna y en el caso de Premium aumenta $4.1 \%$. En promedio, el NO aumentó más para las mezclas de gasolina Magna que para las mezclas de gasolina Premium. Otros investigadores también han observado que el incremento en el NO al adicionar Etanol depende del valor del NO de la gasolina base, obteniéndose

Tabla 2. Valores promedio de octanaje de las mezclas Magna-Etanol y Premium-Etanol

\begin{tabular}{ccccccc}
\hline \multirow{2}{*}{$\begin{array}{c}\text { Concentración } \\
\text { de EtOH } \\
(\% \text { vol. })\end{array}$} & NOI $^{1}$ & NOM $^{2}$ & $\begin{array}{c}\text { Número } \\
\text { de Octano }\end{array}$ & NOI & NOM & $\begin{array}{c}\text { Número } \\
\text { de } \\
\text { Octano }\end{array}$ \\
\cline { 2 - 7 } $0 \%$ & $91.5 \pm 0.2$ & $84.3 \pm 0.1$ & $87.9 \pm 0.1$ & $98 \pm 0.0$ & $87.1 \pm 0.1$ & $92.6 \pm 0.0$ \\
$10 \%$ & $95.6 \pm 0.1$ & $86.1 \pm 0.1$ & $90.8 \pm 0.1$ & $101 \pm 0.0$ & $88.2 \pm 0.1$ & $94.6 \pm 0.0$ \\
$15 \%$ & $97.4 \pm 0.1$ & $86.7 \pm 0.1$ & $92.1 \pm 0.0$ & $102 \pm 0.0$ & $88.4 \pm 0.2$ & $95.2 \pm 0.1$ \\
\hline
\end{tabular}

${ }^{1}$ Número de Octano de Investigación, ${ }^{2}$ Número de Octano del Motor

Tabla 3. Promedio de la Presión de Vapor Seco Equivalente (PVSE) de las mezclas Gasolina-Etanol, reportada en $\mathrm{kPa}$

\begin{tabular}{cccc}
\hline \multirow{2}{*}{ Gasolina Base } & \multicolumn{3}{c}{ EtOH (\% vol) } \\
\cline { 2 - 4 } & $0 \%$ & $10 \%$ & $15 \%$ \\
\hline Magna & $70.4 \pm 0.7$ & $67.5 \pm 1.9$ & $67.2 \pm 0.4$ \\
Premium & $44.7 \pm 1.6$ & $70.3 \pm 1.0$ & $55.3 \pm 4.9$ \\
\hline
\end{tabular}

mayores incrementos para mezclas que emplearon gasolinas base con menor NO (Keller, 1979). Asimismo, se ha observado que la composición de las gasolinas base influye en el valor final del NO (Houben, 1995). Por ejemplo, gasolinas con alto contenido de isoparafinas tienden a aumentar más su NOI y NOM (Anderson et al., 2010). Finalmente, cabe notar que en todos los casos el combustible cumpliría con la especificación establecida por PEMEX Refinación.

\section{Presión de vapor}

La presión de vapor es una propiedad importante de los líquidos volátiles. En la tabla 3 se muestran los valores promedio de la presión de vapor seco equivalente (PVSE) reportados para las muestras analizadas. Se observa que al agregarle Etanol a $10 \%$ a la gasolina Premium, se obtuvo un incremento de más de $50 \%$ en la PVSE (con respecto al combustible sin Etanol) seguido de una disminución en $21 \%$ para la muestra con 15\% Etanol. Sin embargo, para el caso de la gasolina Magna, la PVSE fue máxima para las muestras sin Etanol, obteniéndose una ligera disminución en la mezcla con $10 \%$ Etanol. La diferencia entre las mezclas de gasolina Magna con $10 \%$ y $15 \%$ Etanol fue marginal.

En estudios previos se ha reportado un aumento en la presión de vapor de la gasolina al agregarle un $10 \%$ en volumen de Etanol, seguido de una disminución al aumentar 20\% la concentración de Etanol (Hasan, 2003). Por otra parte, estudios realizados por Furey et al. (1987) revelan que al variar la concentración de Etanol en el rango de $5 \%-10 \%$ se produce un mayor incremento en la presión de vapor, y al incrementar la concentración en el rango de $10 \%-50 \%$ ésta disminuye lentamente mientras que en el rango de $50 \%-100 \%$ decrece rápidamente. Este comportamiento ha sido ratificado en estudios recientes (p. ej., Hatzioannidis et al., 1998; Andersen et al., 2010a). De manera particular, Andersen et al. (2010a) emplearon una gasolina base con PVSE de $61 \mathrm{kPa}$ para investigar el efecto de la adición de Etanol a dicha gasolina. Los resultados obtenidos indicaron un aumento de $11.5 \%$ en la PVSE en su mezcla de gasolina-Etanol al 10\% (v/v), y la PVSE se mantuvo por encima de la de la gasolina base para niveles de Etanol por encima de $45 \%$. 
El Etanol tiene una PVR de $16.0 \mathrm{kPa}$, mientras que las gasolinas base pueden elaborarse en un amplio intervalo de valores de PVR (menores a $50 \mathrm{kPa}$ hasta mayores de 65 $\mathrm{kPa}$ ), dependiendo de la aplicación y consideraciones normativas de cada lugar. En principio, al mezclar gasolina con Etanol no se tiene un comportamiento de una mezcla ideal, es decir, la mezcla no disminuye su PVR proporcionalmente a la cantidad de Etanol que se introduce en la mezcla como sugeriría la Ley de Raoult. Al contrario, las mezclas de gasolina-etanol exhiben un comportamiento no ideal en donde pequeñas cantidades de Etanol ( 5\%-10\%) aumentan la PVR de la mezcla por encima del valor de la gasolina base, para posteriormente comenzar a disminuir. Asimismo, se tiene la formación de una mezcla cuasi-azeotrópica (Andersen et al., 2010b). El comportamiento anterior está relacionado con los puentes de hidrógeno formados entre moléculas de Etanol: la fuerza de estos enlaces mantiene una presión de vapor relativamente baja. Al mezclar la gasolina, que es una mezcla de compuestos no polares, con pequeñas cantidades de Etanol, los puentes de hidrógeno pierden fuerza y el Etanol se comporta como una molécula de bajo peso molecular, más volátil, aumentando así la presión de vapor del sistema (Torres y Molina, 2002). De la misma manera, el alcohol interfiere con las interacciones moleculares entre los hidrocarburos no polares con el mismo efecto de aumentar la presión de vapor (Andersen et al., 2010a). A medida que la cantidad de Etanol aumenta se pudiera argumentar que las moléculas de Etanol vuelven a establecer puentes de hidrógeno con el efecto global de disminuir la presión de vapor de la mezcla.

Un resultado interesante de este trabajo es que, si bien, las mezclas de gasolina Premium con Etanol exhibieron en lo general el comportamiento no ideal descrito arriba, las mezclas de gasolina Magna con Etanol no lo hicieron. Este hecho puede atribuirse a que se ha encontrado que el comportamiento de la PVR en mezclas gasolina-Etanol es función de la composición de la gasolina empleada (Andersen et al., 2010a). Es más, se ha reportado que la PVR llega a incrementarse más cuando se mezcla Etanol con gasolinas base con menor PVR (American Petroleum Institute, 2001), como fue el caso observado aquí con la gasolina Premium. Del mismo modo, se pudiera esperar que el incremento fuera menor con gasolinas base con mayor PVR (como el caso de la gasolina Magna). Esto último tendría que explorarse con mayor detalle.

\section{Curva e índice de destilación}

El Etanol, como todo compuesto en su estado puro, tiene una temperatura única de ebullición a una presión dada $\left(78.3^{\circ} \mathrm{C}\right.$ a $1 \mathrm{~atm}$ de presión), mientras que la gasolina por ser una mezcla de hidrocarburos tiene un intervalo de temperaturas de ebullición a una presión dada $\left(30^{\circ} \mathrm{C}-200^{\circ} \mathrm{C}\right.$ a $1 \mathrm{~atm}$ de presión). Dicho intervalo es una función de la composición de la gasolina, y típicamente se representa por una curva de destilación. La curva de destilación brinda información acerca de la composición, las propiedades y el comportamiento del combustible durante su almacenamiento y uso (Bruno et al., 2009). La $T_{10}$ (temperatura a la cual $10 \%$ de la muestra se ha evaporado) representa la capacidad del combustible para evaporarse rápidamente a bajas temperaturas, mientras que $T_{50}$ y $T_{90}$ representan la habilidad de los componentes pesados presentes en el combustible para evaporarse conforme el motor se calienta y para ser quemados. La $T_{10}$ debe ser suficientemente baja como para proporcionar un fácil arranque en frío y tan alta como para minimizar los tapones de vapor; la $T_{50}$ debe ser suficientemente baja como para poder alcanzar sin problemas la temperatura de régimen y proporcionar un buen desempeño en climas fríos, sin ser tan baja que provoque problemas de sobrecalentamiento y tapones de vapor (Bruno et al., 2009). La $T_{90}$ y la Temperatura Final de Ebullición (TFE) deben ser suficientemente bajas para minimizar depósitos en la cámara de combustión (Owen y Coley, 1995).

Como se comentó con anterioridad, la adición de pequeñas cantidades de Etanol a la gasolina aumenta la presión de vapor y, por ende, disminuye la temperatura de ebullición de las especies de hidrocarburos presentes. Los compuestos alifáticos son más susceptibles a este efecto que los aromáticos (Gerdes y Suppes, 2001). La figura 1 muestra las curvas de destilación obtenidas para mezclas de ambas gasolinas. Las principales variaciones en las curvas de destilación corresponden al valor de la $T_{50}$. Por un lado, la $T_{50}$ de las muestras Magna E10 y E15 disminuye en promedio un 21.6\% y $22.0 \%$, respectivamente, con respecto a la gasolina sin Etanol. Por otro lado, la $T_{50}$ de las muestras de Premium E10 y E15 disminuye en un 15.9\% y 13.9\%, respectivamente. Este cambio en la $T_{50}$ se debe al efecto de la adición de Etanol en el punto de ebullición de los alifáticos, y es acorde a lo que otros investigadores han reportado para mezclas gasolina-etanol en donde se ha apreciado que los principales cambios en las curvas de destilación ocurren entre $T_{10} \mathrm{y}_{40}$ (Andersen et al., 2010b; Furey y Perry, 1987; Greenfield et al., 1998). Los valores de $T_{90} \mathrm{y}$ la temperatura final de ebullición (TFE) no se ven alterados significativamente ni para las mezclas con gasolina Magna, ni para las mezclas con gasolina Premium por ser este el intervalo de ebullición de los compuestos aromáticos (variación no mayor a $3.1 \%$, respecto a la 


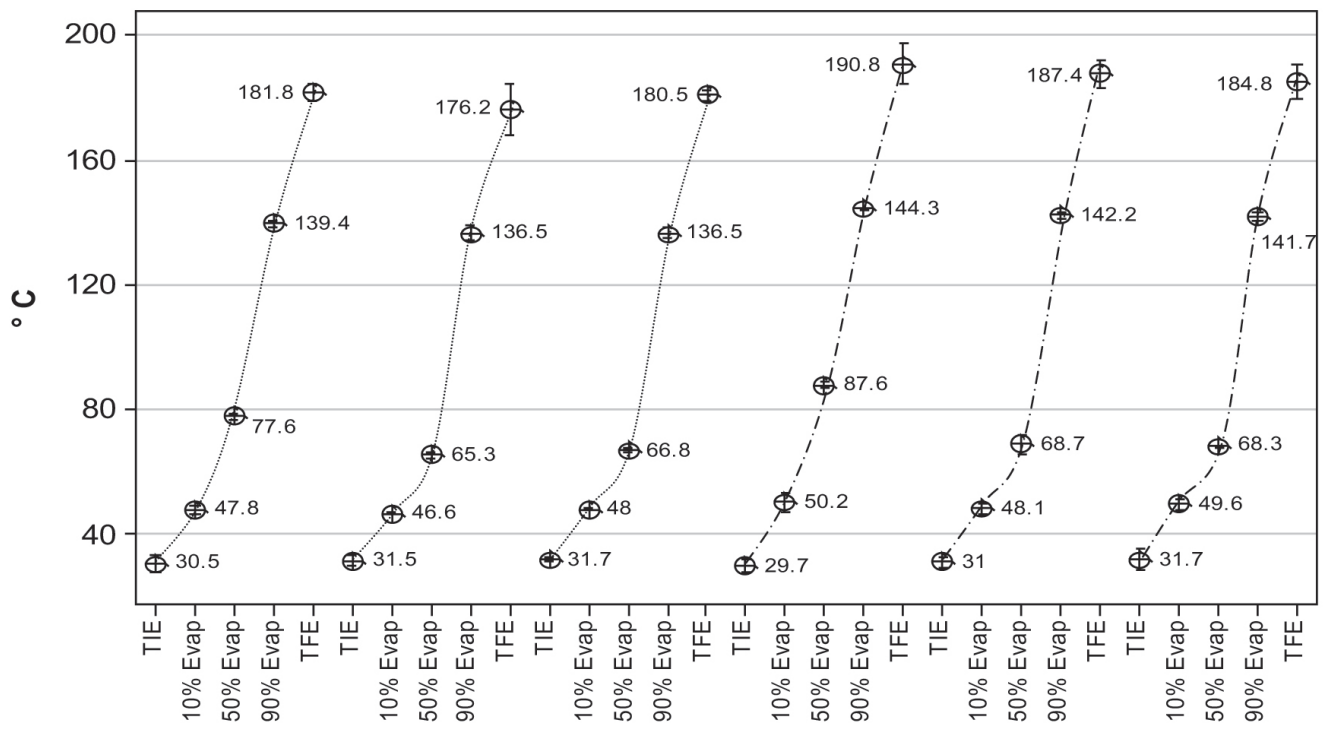

PREMIUM PREMIUM-10\%EtOH PREMIUM-15\%EtOH
Figura 1. Curvas de destilación de la gasolina Premium y Magna reformulada con Etanol (los indicadores de incertidumbre representan intervalos de confianza de 95\%) gasolina base). Andesen et al. (2010b) también observaron reducciones de solamente $2^{\circ} \mathrm{C}$ a $4^{\circ} \mathrm{C}$ de $T_{60}$ en adelante en sus muestras de E10. De igual manera, el cambio en la temperatura inicial de ebullición (TIE) y la $\mathrm{T}_{10}$ al agregar Etanol es relativamente bajo en todos los casos. Este mismo comportamiento fue observado por Andersen et al. (2010b). La TIE es mayor en las muestras $\mathrm{E}_{10} \mathrm{y}_{15}$, tanto para las mezclas con gasolina Magna (4.4\% y $6.7 \%$, respectivamente) como para las mezclas con gasolina Premium (3.3\% y 3.9\%, respectivamente), respecto a la gasolina base. Ya para la $\mathrm{T}_{10}$, las mezclas con Etanol tienden a tener un valor marginalmente inferior que las muestras de gasolina base.

Así, la curva de destilación es útil para determinar el índice de destilación, que es una medida de la volatilidad de la gasolina, primordialmente de su tendencia a vaporizarse en el motor durante el arranque inicial y calentamiento del mismo. El límite para el índice de destilación en Estados Unidos no debe exceder los $1,250^{\circ} \mathrm{F}\left(676.6^{\circ} \mathrm{C}\right)$. En el estándar ASTM D-4814 se encuentra la ecuación para calcularlo:

$I D=1.5 T_{10}+3 T_{50}+T_{90}+2.4 V$

donde $V$ es el porcentaje de etanol en volumen presente en la mezcla y las $T_{10}, T_{50}$ y $T_{90}$ están en grados Fahrenheit.

En la figura 2 se reportan los índices de destilación calculados. Con respecto a las gasolinas base, el índice de destilación disminuye poco más de $8 \%$ y $5 \%$ para gasolina Magna y Premium con Etanol a 10\% en volu- men, respectivamente. Se ha notado que conforme el índice de destilación aumenta, la presión de vapor disminuye (Maples, 2000). Esto concuerda con los resultados de presión de vapor obtenidos para gasolina Premium (figura 2).

\section{Poder calorífico}

El promedio del poder calorífico de las tres repeticiones realizadas a las muestras de gasolina Premium fue de $44.5 \mathrm{MJ} / \mathrm{kg}$, el de Magna correspondió a un valor de 43.4 MJ/kg, mientras que el del Etanol es $27 \mathrm{MJ} / \mathrm{Kg}$ (tabla 4). Por lo tanto, una de las principales desventajas reconocidas que representa el uso de Etanol como combustible es su relativamente bajo poder calorífico. Así, el poder calorífico de las mezclas disminuye con la adición de Etanol. Si las gasolinas mexicanas se llegaran a oxigenar con Etanol a $15 \%$ en volumen, el rendimiento del combustible (expresado como $\mathrm{km}$ recorridos/L) de las mismas disminuiría teóricamente un $8.1 \%$ y $5.1 \%$ para Magna y Premium, respectivamente (vea el apéndice).

De acuerdo con el balance energético nacional para el 2005, hubo un consumo de 1,194.05 PJ, por parte del sector autotransporte (SENER, 2006), equivalente a $27.6 \mathrm{Tg}$ de gasolina Manga (según el poder calorífico reportado en la tabla 4), que después de su combustión hubieran liberado a la atmósfera aproximadamente 85.2 Tg de $\mathrm{CO}_{2}$ (vea el apéndice). Para este análisis de escenarios se supone que toda la gasolina empleada fue Magna. Al reformular el combustible (gasolina Magna) con 10\% de Etanol, teóricamente se necesitarían 28.84 Tg de 


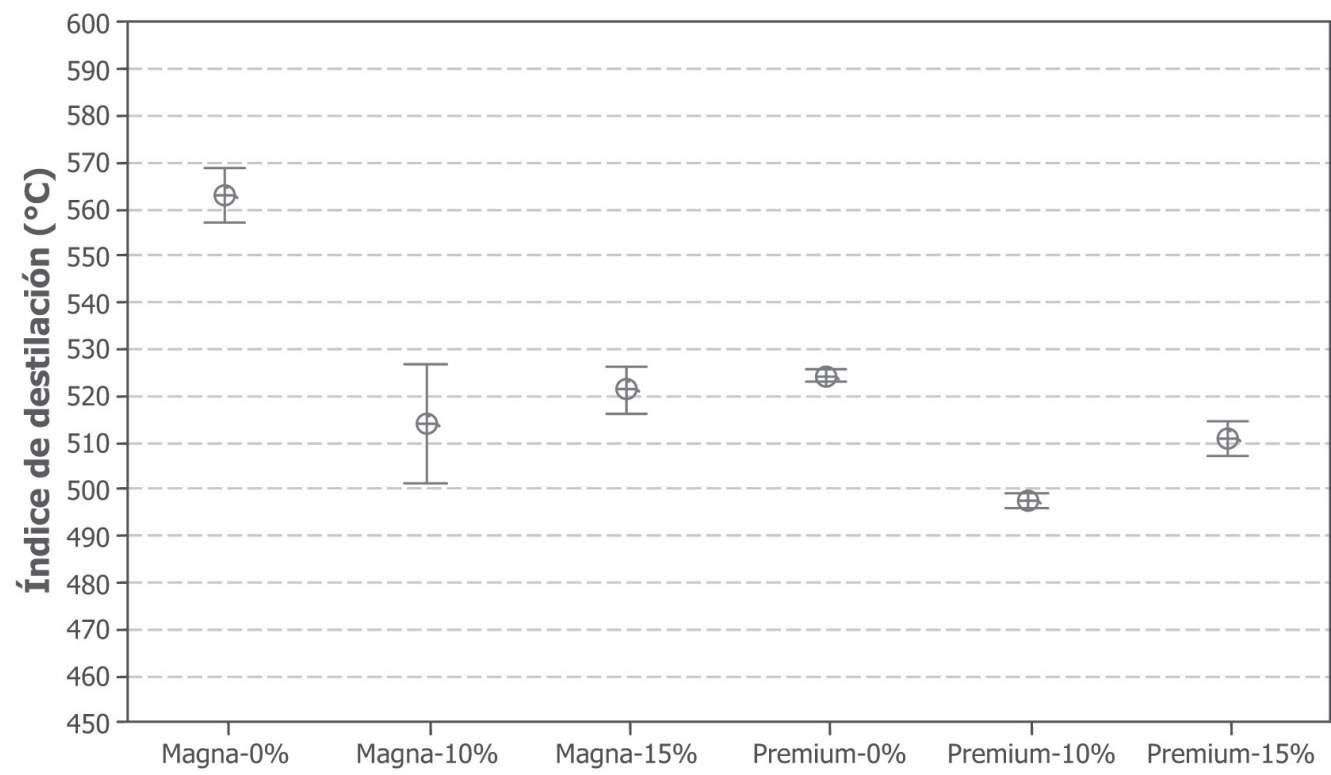

Figura 2. Índice de destilación calculado para la gasolina Magna y Premium reformulada con Etanol (las barras de error representan intervalos de confianza de $95 \%)$ combustible para liberar los mismos 1,194.050 PJ; mientras que si se reformula el mismo combustible con Etanol a $15 \%$, en teoría $30.62 \mathrm{Tg}$ de combustible son los necesarios para obtener la misma cantidad de energía (ambos valores empleando, nuevamente, los poderes caloríficos reportados en la tabla 4). En cuanto a emisiones teóricas de $\mathrm{CO}_{2}$, añadir Etanol al 10\% equivale a la emisión de $85.0 \mathrm{Tg}$ de $\mathrm{CO}_{2}$, de los cuales $7.0 \%$ proviene del Etanol (apéndice). Las emisiones provenientes de fuentes fósiles ascenderían a 79.05 Tg. En el caso de la gasolina Magna reformulada con Etanol a 15\%, las emisiones ascenderían a $88.3 \mathrm{Tg}$ de $\mathrm{CO}_{2}$ de los cuales $10.7 \%$ provendrían de una fuente renovable. En resumen, al reformular la gasolina con Etanol a 10\% las emisiones netas (emisiones fósiles exclusivamente) de $\mathrm{CO}_{2}$ disminuirían en un $7.2 \%$ y con Etanol a $15 \%$ presentan un decremento de $7.5 \%$, con respecto a las emisiones de la gasolina sin Etanol. El beneficio en emisiones de $\mathrm{CO}_{2}$ entre las gasolinas E10 y E15 es marginal por la pérdida

Tabla 4. Promedio del poder calorífico de los combustibles analizados

\begin{tabular}{cccc}
\hline \multirow{2}{*}{$\begin{array}{c}\text { Concentración de } \\
\text { EtOH (\% vol.) }\end{array}$} & \multicolumn{3}{c}{ Poder calorífico (MJ/Kg) } \\
\cline { 2 - 4 } & Diesel & Magna & Premium \\
\hline $0 \%$ & $45.0 \pm 0.1$ & $43.3 \pm 0.1$ & $44.5 \pm 0.4$ \\
$10 \%$ & $43.1 \pm 0.5$ & $41.4 \pm 0.5$ & $41.7 \pm 0.4$ \\
$15 \%$ & $42.7 \pm 0.3$ & $39.0 \pm 0.5$ & $41.4 \pm 0.4$ \\
\hline
\end{tabular}

de poder calorífico en la mezcla de E15 que resulta en un incremento importante de consumo de combustible. Estos últimos cálculos no toman en consideración el $\mathrm{CO}_{2}$ liberado por el Etanol, debido a que por provenir de un insumo agrícola el $\mathrm{CO}_{2}$ liberado se compensa con el absorbido por las plantas durante su crecimiento. Asimismo, los cálculos no consideran eficiencias de combustión (apéndice), ya que la acentuación aquí no es en la masa total emitida, sino en el cambio porcentual que se pudiera llegar a obtener al sustituir el uso de un combustible por otro (suponiendo misma eficiencia de combustión).

\section{Resultados para las mezclas Diesel-Etanol}

\section{Estabilidad de las mezclas}

Estudios previos han demostrado que al mezclar diesel con Etanol en proporciones superiores de 3\% a 5\% en volumen de Etanol se requieren aditivos (cosolventes) para evitar separación de fases en la mezcla (Gerdes y Suppes, 2001; Reyes et al., 2009). En el caso se las mezclas de diesel con Etanol trabajadas en este estudio, todas fueron inestables incluso a temperatura ambiente, por lo que fue necesario utilizar aditivos. Se evalúo el desempeño de los dos aditivos mencionados en la sección de metodología, obteniéndose buenos resultados con ambos. Sin embargo, al realizar la prueba de poder calorífico se observó que la mezcla que contenía biodiesel como aditivo tenía un valor mayor, por tanto se optó por utilizar el biodiesel como aditivo. Otro punto a fa- 
vor de usar la mezcla diesel-biodiesel-Etanol es que se ha encontrado que la adición de biodiesel compensa en parte la reducción en el Número de Cetano y la lubricidad derivada de la adición del Etanol (Lapuerta et al., 2009). La concentración de biodiesel fue de $5 \%$ y $10 \%$, para las mezclas con Etanol a $10 \%$ y $15 \%$, respectivamente. Otros autores recomiendan relaciones 1:1 de Etanol a Biodiesel, aunque dicha selección depende del combustible base empleado en las mezclas (Fernando y Hanna, 2004).

\section{Caracterización fisicoquímica}

El Número de Cetano (NC) es tal vez el parámetro más importante para caracterizar un combustible que se emplea en un motor diesel (Ribeiro et al., 2007). El NC mide qué tan propensa es una mezcla combustible de llegar a su punto de autoignición al ser suministrada al motor. En principio, entre mayor sea el valor del NC el desempeño del motor mejora, aunque valores superiores a 60 ya no proveen beneficios significativos (Gerdes y Suppes, 2001). El diesel que se utilizó como base para hacer las mezclas con Etanol reportadas en este trabajo tiene un NC de 52, aproximadamente (lo cual se considera un valor más que aceptable; p. ej., Gerdes y Suppes, 2001). Al adicionar Etanol (NC alrededor de 8) el valor del NC de la mezcla disminuyó, obteniéndose valores parecidos para las mezclas con $10 \%$ y $15 \%$ de Etanol (tabla 5). La reducción del NC por la adición del Etanol se debe a que es una molécula pequeña que tiene alta resistencia a pasar por un proceso de escisión por radicales libres, a que los grupos funcionales de los alcoholes tienden a generar bajos NC y a que su alto calor latente de vaporización tiende a enfriar la carga de combustible durante el proceso de inyección de combustible al motor (Gerdes y Suppes, 2001). En contraste, el biodiesel está compuesto de moléculas más grandes que por sus características químicas tiende a tener NC entre 48 y 67 (Ribeiro et al., 2007). Así, la mayor disminución del NC para la mezcla a 10\% de Etanol estaría explicada por el hecho de que en esta mezcla la proporción Etanol-Biodiesel fue mayor que en la de 15\% Etanol (2:1 vs 1.5:1, respectivamente), por lo que la presencia de Biodiesel estaría en efecto compensando el efecto de reducción del NC provocado por la presencia del Etanol. Finalmente, el NC obtenido de todas las mezclas estuvo por encima de un valor de 45 que es el valor recomendado para aprovechar el efecto que la adición de Etanol tiene en la reducción de emisiones de NOx y partículas suspendidas finas (Gerdes y Suppes, 2001).

El punto de inflamabilidad es la temperatura a la cual existe una tendencia a formar una mezcla combus- tible con el aire. Es una propiedad de importancia en el diesel y es deseable que ésta no sea muy baja por razones de seguridad. Se observó que al agregar Etanol, el punto de inflamabilidad de las muestras tiende a disminuir y la mezcla con $10 \%$ de Etanol es la que formó una mezcla flamable a menor temperatura (tabla 5). La obtención de un mínimo con 10\% de Etanol nuevamente puede explicarse por las proporciones de Etanol-Biodiesel que se usaron en las mezclas a $10 \%$ y $15 \%$. El biodiesel, al ser una mezcla con un muy alto punto de inflamabilidad estaría compensando la reducción provocada por la adición del Etanol (Ribeiro et al., 2007). Por otro lado, se observó que el punto inicial de ebullición y la $T_{10}$ de las mezclas, disminuyeron considerablemente con la adición de Etanol (un compuesto con un punto de ebullición significativamente menor que el combustible base), y en ambos casos (al 10\% y 15\% en volumen) no se alcanzó a recuperar $90 \%$ de la mezcla, posiblemente debido a la presencia del biodiesel del cual tiene Flash Points muy superiores al del diesel (tabla 6).

Tabla 5. Valores promedio para el Número Cetano y el punto de inflamabilidad del diesel reformulado con Etanol

\begin{tabular}{ccc}
\hline \multirow{2}{*}{$\begin{array}{c}\text { Concentración } \\
\text { de Etanol } \\
(\% \text { vol })\end{array}$} & Número de Cetano & $\begin{array}{c}\text { Punto de } \\
\text { Inflamabilidad }\left({ }^{\circ} \mathrm{C}\right)\end{array}$ \\
\cline { 2 - 3 } $0 \%$ & $52.6 \pm 0.5$ & $53.9 \pm 1.6$ \\
$10 \%$ & $45.6 \pm 1.0$ & $23.3 \pm 1.7$ \\
$15 \%$ & $45.8 \pm 1.8$ & $26.9 \pm 1.1$ \\
\hline
\end{tabular}

\section{Poder calorífico}

Para el caso del poder calorífico del Diesel-Etanol ocurrió lo mismo que con la gasolina: éste disminuye con la adición de Etanol. La mayor reducción del poder calorífico es con $15 \%$ en volumen de Etanol añadido (tabla 4). El poder calorífico del biodiesel empleado como aditivo en las mezclas diesel-Etanol no fue caracterizado; sin embargo, caracterizaciones previas del poder calorífico del biodiesel obtenido en la misma planta piloto marca un intervalo de valores entre 40.8 y $43.4 \mathrm{MJ} / \mathrm{Kg}$ (Gutiérrez, 2006).

El consumo energético nacional aportado por el diesel ascendió a 437.80 PJ en el 2005 (SENER, 2006), lo cual representaría teóricamente el consumo de $9.73 \mathrm{Tg}$ de diesel (empleando el valor del poder calorífico reporta- 
Tabla 6. Valores promedio de la destilación de las mezclas Diesel-Etanol

\begin{tabular}{cccc}
\hline & $\begin{array}{c}0 \% \text { vol. } \\
\text { EtOH }\end{array}$ & $\begin{array}{c}10 \% \text { vol. } \\
\text { EtOH }\end{array}$ & $\begin{array}{c}15 \% \text { vol } \\
\text { EtOH }\end{array}$ \\
\hline $\mathrm{TIE}^{1},{ }^{\circ} \mathrm{C}$ & $175.8 \pm 1.6$ & $65.7 \pm 21.0$ & $78.0 \pm 0.1$ \\
$10 \%$ Evap,${ }^{\circ} \mathrm{C}$ & $200.1 \pm 1.4$ & $112.6 \pm 53.8$ & $79.5 \pm 2.3$ \\
$50 \%$ Evap, ${ }^{\circ} \mathrm{C}$ & $267.5 \pm 0.3$ & $256.5 \pm 2.3$ & $258.2 \pm 0.9$ \\
$90 \%$ Evap, ${ }^{\circ} \mathrm{C}$ & $336.3 \pm 1.2$ & --- & --- \\
$\mathrm{TFE}^{2},{ }^{\circ} \mathrm{C}$ & $357.5 \pm 3.0$ & $354.1 \pm 6.9$ & $349.4 \pm 12.3$ \\
Recuperado, $\%$ & $98.2 \pm 0.3$ & $89.4 \pm 4.1$ & $86.4 \pm 0.9$ \\
Residuo, $\%$ & $0.8 \pm 0.4$ & $10.5 \pm 4.1$ & $13.5 \pm 0.9$ \\
Pérdida, $\%$ & $1.0 \pm 0.2$ & $0.1 \pm 0.0$ & $0.1 \pm 0.0$ \\
\hline
\end{tabular}

${ }^{1}$ TIE: Temperatura Inicial de Ebullición,

${ }^{2}$ Temperatura Final de Ebullición

do en la tabla 4). Las emisiones teóricas de $\mathrm{CO}_{2}$ derivadas de este consumo energético ascienden a $30.74 \mathrm{Tg}$ (ver Apéndice). Si se reformulara el diesel con Etanol a $10 \%$, las emisiones teóricas ascenderían a $30.96 \mathrm{Tg}$ de $\mathrm{CO}_{2}$. Sin embargo, $1.85 \mathrm{Tg}$ de ese $\mathrm{CO}_{2}$ no provendría del combustible fósil. Por tanto, las emisiones con respecto al diesel sin reformular disminuirían en un $5.3 \%$. Por otro lado, si el contenido de Etanol se incrementa a 15\% en volumen, las emisiones ascenderían a $30.74 \mathrm{Tg}$, de los cuales $9.2 \%$ no provendrían del combustible fósil y, por tanto, con respecto al diesel sin reformular, las emisiones disminuirían en un 9.2\%. Al igual que en el caso de los cálculos para las mezclas de gasolina, estos cálculos para las mezclas de diesel no consideran la eficiencia de combustión al ser el énfasis en el cambio porcentual de emisiones entre el uso de mezclas y no en la masa total emitida.

\section{Estabilidad a la oxidación}

El periodo de inducción puede ser usado como un indicador de la tendencia de la gasolina a formar gomas durante el periodo de almacenamiento. Esta prueba se incluyó en el presente estudio con la finalidad de analizar el efecto del Etanol añadido a la gasolina, respecto a la formación de gomas. La estabilidad a la oxidación se determinó por el método de periodo de inducción (ASTM D-525). La formación de gomas es una característica indeseable en las gasolinas, ya que un nivel elevado de las mismas en el combustible llega a dañar seriamente el motor de un vehículo.

Para todas las muestras se obtuvo el mismo resultado: el periodo de inducción reportado fue mayor a 1440 minutos. Las especificaciones para gasolinas mexicanas establecen un valor de al menos 300 minutos (PEMEX Refinación, 2008), por lo tanto, las gasolinas cumplen con la especificación. Pereira y Pasa (2005) también realizaron pruebas de estabilidad a la oxidación de gasolina con Etanol en un intervalo de 0\% a 30\% Etanol en volumen. Dichos investigadores demostraron que la adición de Etanol no causó ni un incremento, ni una reducción en el contenido de gomas, y tampoco se comportó como un catalizador o inhibidor de la oxidación de olefinas presentes. Asimismo, indican que una posible explicación de este resultado es que el mecanismo de oxidación del Etanol no es radicalar, mientas que el de la oxidación de olefinas sí lo es. En este proceso, el Etanol se oxida a aldehído y posteriormente a ácido carboxílico sin formar gomas en el proceso. También se ha argumentado que agregar Etanol a las gasolinas pudiera incluso ser benéfico a los motores al reducir la deposición de gomas por litro de gasolina consumida, debido al aparente efecto de dilución de las gomas derivadas de la gasolina (Pereira y Pasa, 2005).

\section{Conclusiones}

Las pruebas realizadas corroboraron que el Etanol es un buen potenciador del octanaje. En las muestras estudiadas, el Número de Octano reportado aumentó hasta en un $6.5 \%$ con la adición de Etanol a 15\% en volumen a la gasolina Magna. Así mismo, la presión de vapor aumentó en poco más de $50 \%$ al adicionar 10\% de Etanol a la gasolina Premium. Esto implicaría un aumento en las emisiones evaporativas de no aplicarse un control apropiado a los sistemas de suministro de la gasolina a los vehículos automotor. La $T_{50}$ disminuyó hasta en $20 \%$ aproximadamente; esta disminución podría provocar problemas relacionados con la formación de tapones de vapor. El rendimiento promedio del combustible expresado en $\mathrm{km} / \mathrm{L}$, con base en los datos de poder calorífico obtenidos, disminuiría hasta en un $8.1 \%$ en gasolina Magna oxigenada con Etanol a 15\%. En cuanto a las emisiones teóricas de $\mathrm{CO}_{2}$, al reformular la gasolina Magna con Etanol a 10\% disminuirían en $7.2 \%$ y con Etanol a $15 \%$ presentarían un decremento de $7.5 \%$, con respecto a las de la gasolina sin Etanol. Lo anterior sin tomar en consideración el $\mathrm{CO}_{2}$ liberado por la combustión del Etanol. En cuanto a las mezclas diesel-Etanol, el Número de Cetano de las mezclas disminuyó hasta $13.0 \%$ y su poder calorífico disminuyó en $2.3 \mathrm{MJ} / \mathrm{Kg}$ si éste se oxigena con $15 \%$ de Etanol. En cuanto a las emisiones teóricas de $\mathrm{CO}_{2}$, con Etanol a 10\%, éstas disminuyeron 5.3\% y con Etanol a 15\% presentaron un decremento de $9.2 \%$. 


\section{Apéndice}

Cambio estimado en el rendimiento de las mezclas gasolina-etanol

A las mezclas gasolina-Etanol se les determinó su densidad a partir del método ASTM D 218 (Castillo, 2008), los valores obtenidos se presentan en la tabla A.1. Así, es posible calcular el poder calorífico base volumen de cada uno de estos combustibles, de acuerdo a dichos valores de densidad y el poder calorífico reportado en la tabla 4 (tabla A.1). Considerando que la eficiencia energética es la misma empleando la gasolinas base y sus contrapartes de mezclas gasolina-Etanol, las diferencias porcentuales entre los valores del poder calorífico base volumen presentadas en la tabla A.1 representarían una aproximación a la diferencia del desempeño del rendimiento de combustible en términos de $\mathrm{Km}$ recorridos por litro de combustible quemado.
$5.96 \mathrm{Tg} \mathrm{CO}_{2}$ (7.0\% del total de $\mathrm{CO}_{2}$ teórico). Con el mismo procedimiento y suposiciones se estiman las emisiones teóricas de $\mathrm{CO}_{2}$ para una mezcla de Magna E15.

Estimación de emisiones de $\mathrm{CO}_{2}$ : mezclas diesel-Etanol

La densidad del diesel y las mezclas diesel-Etanol fueron determinadas de acuerdo al estándar ASTM D-1298, obteniéndose los siguientes resultados $\left(\mathrm{a} 20^{\circ} \mathrm{C}\right)$ :

1) Diesel $836.7 \pm 7.1 \mathrm{Kg} / \mathrm{L}$,

2) Diesel E10 830.0 $\pm 0.0 \mathrm{Kg} / \mathrm{L}$,

3) Diesel E15 825.0 $\pm 0.0 \mathrm{Kg} / \mathrm{L}$ (el valor de incertidumbre se presenta como nivel de confianza a $95 \%$ ).

Considerando al diesel como una mezcla de hidrocarburos con peso molecular promedio de $211.7 \mathrm{~g} / \mathrm{gmol}$, $86.13 \%$ en peso de $\mathrm{C}$ y $13.87 \%$ en peso de $\mathrm{H}$ (Lapuerta et

Tabla A.1. Densidad (a $20^{\circ} \mathrm{C}$ ) y poder calorífico (base volumen) de gasolinas base y las correspondientes mezclas gasolina-Etanol

\begin{tabular}{|c|c|c|c|c|c|c|}
\hline \multirow[b]{2}{*}{$\begin{array}{l}\text { Concentración de } \\
\text { Etanol (\%vol) }\end{array}$} & \multicolumn{3}{|c|}{ Magna } & \multicolumn{3}{|c|}{ Premium } \\
\hline & $\begin{array}{c}\text { Densidad } \\
(\mathrm{Kg} / \mathrm{L})\end{array}$ & $\begin{array}{l}\text { Poder calorífico } \\
\text { (MJ/L) }\end{array}$ & $\begin{array}{c}\text { Diferencia en PC } \\
\%^{\mathrm{a}}\end{array}$ & $\begin{array}{c}\text { Densidad } \\
(\mathrm{Kg} / \mathrm{L})\end{array}$ & $\begin{array}{l}\text { Poder calorífico } \\
\text { (MJ/L) }\end{array}$ & $\begin{array}{c}\text { Diferencia en PC } \\
\%\end{array}$ \\
\hline $0 \%$ & $720.0 \pm 0.0^{\mathrm{b}}$ & 31,176 & - & $705.7 \pm 1.5$ & 31,404 & - \\
\hline $10 \%$ & $730.0 \pm 0.0$ & 30,222 & $-3.1 \%$ & $715.0 \pm 0.0$ & 29,816 & $-5.1 \%$ \\
\hline $15 \%$ & $734.3 \pm 2.9$ & 28,637 & $-8.1 \%$ & $720.0 \pm 0.0$ & 29,808 & $-5.1 \%$ \\
\hline
\end{tabular}

${ }^{a}$ Diferencia en poder calorífico base volumen entre la mezcla gasolina-Etanol respecto a la gasolina base correspondiente.

${ }^{\mathrm{b}}$ La incertidumbre se presenta como nivel de confianza a 95\%.

Estimación de emisiones de $\mathrm{CO}_{2}$ : mezclas gasolina-Etanol

Considerando a la gasolina Manga como $\mathrm{C}_{8} \mathrm{H}_{18}-\mathrm{PM}$ 114 g/gmol- (Anderson et al., 2010; Turns, 2000), la combustión de $27.6 \mathrm{Tg}$ de gasolina equivaldría a la emisión teórica de 85.2 $\mathrm{Tg} \mathrm{CO}_{2}$. Para una mezcla de Magna E10, se requerirían $39.51 \times 10^{6} \mathrm{~L}$ de combustible para liberar 1,194.05 PJ, según los valores de la tabla A.1. Suponiendo una mezcla ideal entre la gasolina y el Etanol, al tener una mezcla a $10 \%$ volumen de Etanol, representaría $35.56 \times 10^{6} \mathrm{~L}$ de gasolina Magna y $39.51 \times 10^{5} \mathrm{~L}$ de Etanol, o bien, 25.60 Tg de gasolina Magna (según la densidad reportada en la tabla A.1) y $3.12 \mathrm{Tg}$ de Etanol (densidad de $789 \mathrm{Kg} / \mathrm{L} \mathrm{a} 20^{\circ} \mathrm{C}$ ). Entonces, las emisiones teóricas de $\mathrm{CO}_{2}$ por la combustión de la gasolina serían $79.05 \mathrm{Tg}$ $\mathrm{CO}_{2}$, y para el caso del Etanol (PM $46 \mathrm{~g} / \mathrm{gmol}$ ) serían de al., 2010), se puede representar al diesel con una fórmula química equivalente a $\mathrm{C}_{15.2} \mathrm{H}_{29.3}$. Las emisiones teóricas de $\mathrm{CO}_{2}$ derivadas de la combustión de este energético ascenderían a $30.74 \mathrm{Tg}$. Para una mezcla Diesel E10, se requerirían $1.22 \times 10^{7} \mathrm{~L}$ de combustible para liberar 437.80 PJ, según los valores de densidad obtenidos. Suponiendo una mezcla ideal entre el diesel y el Etanol, al tener una mezcla a 10\% volumen de Etanol, esto representaría $1.10 \times 10^{7} \mathrm{~L}$ de diesel y $1.22 \times 10^{6} \mathrm{~L}$ de Etanol, o bien, $9.22 \mathrm{Tg}$ de diesel y $0.97 \mathrm{Tg}$ de Etanol. Entonces, las emisiones teóricas de $\mathrm{CO}_{2}$ por la combustión de la gasolina serían $29.11 \mathrm{Tg} \mathrm{CO}_{2}$ y para el caso del Etanol serían de $1.85 \mathrm{Tg} \mathrm{CO}_{2}$. $(6.0 \%$ del total de $\mathrm{CO}_{2}$ teórico). Con el mismo procedimiento y suposiciones se estiman las emisiones teóricas de $\mathrm{CO}_{2}$ para una mezcla de Diesel E15. 


\section{Agradecimientos}

Los autores agradecen el apoyo recibido por el CONACYT y el Gobierno del Estado de Nuevo León a través de su programa de Fondos Mixtos para la realización de este estudio. Apoyo complementario para el estudio fue otorgado por el Tecnológico de Monterrey a través de su programa de Cátedras de Investigación (CAT-186).

\section{Referencias}

American Petroleum Institute. Alcohols and Ethers: A Technical Assessment of Their Application as Fuels and Fuel Components, $3^{\text {rd }}$ ed., API publication 4251, Washington, DC, 2001.

Andersen V.F., Anderson J.E., Wallington T.J., Mueller S.A. y Nielsen O.J. Vapor Pressures of Alcohol-Gasoline Blends. Energy Fuels, volumen 24, 2010a:3647-3654.

Andersen V.F., Anderson J.E., Wallington T.J., Mueller S.A. y Nielsen O.J. Distillation Curves for Alcohol-Gasoline Blends. Energy Fuels, volumen 24, 2010b :2683-2691.

Ardenson J.E., Kramer U., Mueller S.A. y Wallington T.J. Octane Numbers of Ethanol and Methanol-Gasoline Blends Estimated from Molar Concentrations. Energy Fuels, volumen 24, 2010:6576-6585.

Arnould A. Additifs Permettant l'Incorporation d'Alcools Aux Carburantes Renfermant Lesdits Additifs. Institut National de la Propriété Industrielle. Int. C10L 1/18. 1981-03-02. Societe d'Etudes et de Realisations No. 8104112, 1982.

Bruno T.J., Wolk A. y Naydich A. Composition-Explicit Distillation Curves for Mixtures of Gasolina with Four-Carbon Alcohols (Butanols). Energy \& Fuels, volumen 23, 2009:2295-2306.

Cleveland C. Net Energy from the Extraction of Oil and Gas in the United States. Energy, volumen 30, 2005:769-782.

Farrell A.E., Plevin R.J., Turner B.T., Jones A.D., O'Hare M. y Kammen, D.M. Ethanol can Contribute to Energy and Environmental Goals. Science, volumen 311, 2006:506-508.

Fernando S. y Hanna M. Development of a Novel Biofuel Blend Using Ethanol-Biodiesel-Diesel Microemulsions: EB-Diesel. Energy \& Fuels, volumen 18, 2004:1695-1703.

French R. y Malone P. Phase Equilibria of Etanol fuel Blends. Fluid Phase Equilibria, volúmenes 228-229, 2005:27-40.

Furey R.L. y Perry K.L. Vapor Pressure of Mixtures of Gasolines and Gasoline-Ethanol Blends. Society of Automotive Engineers, volumen 10, 1987:1-12.

Gerdes K.R. y Suppes G.J. Miscibility of Ethanol in Diesel Fuels. Ind. Eng. Chem. Res., volumen 40, 2001:949-956.

Gnansounou E. y Dauriat A. Energy Balance of Bioethanol: A Synthesis. European Biomass Conference and Exhibition, Paris, Francia, 2005.
Gramajo de Doz M.B., Bonatti C.M. y Sólimo, H.N. Water Tolerance and Ethanol Concentration in Ethanol-Gasoline Fuels at three Temperatures. Energy and Fuels, volmen 18, 2004:334-337.

Greenfield M.L., Lavoie G.A., Smith C.S. y Curtis E.W. Technical Paper Series, 982724; Society of Automotive Engineers, 1998.

Gutierrez-Gómez F. Estudio preliminar para la optimización y análisis económico para la producción de biodiesel en la planta piloto del ITESM a partir de aceite vegetal usado, tesis (maestría), México, Tecnológico de Monterrey, Monterrey, 2007, 119 p.

Hatzioannidis I., Voutsas E.C., Lois E. y Tassios D.P. Measurement and Prediction of Reid Vapor Pressure of Gasoline in the Presence of Additives. J. Chem. Eng. Data, volumen 43, 1998:386-392.

Hasan M. Effect of Ethanol-Unleaded Gasoline Blends on Engine Performance and Exhaust Emission. Energy Conversion and Management, volumen 44, 2003:1547-1561.

Hill J., Nelson E., Tilman D., Polasky S. y Tiffany D. Environmental, Economic, and Energetic Costs and Benefits of Biodiesel and Ethanol Biofuels. Proceedings of the National Academy of Sciences of the United States of America, volumen 103, 2006:11206-11210.

Houben M.C.M. Oxygenated Blending Components for GasolineAlcohols and Ethers, en: Motor Gasoline. Marshall E.L. y Owen K., eds. The Royal Society of Chemistry, Cambridge, UK, 1995.

INE (Instituto Nacional de Ecología). 2006. Inventario nacional de gases de efecto invernadero 1990-2002, México, pp. 16-18.

IPCC (Intergovernmental Panel on Climate Change). 2001. Climate Change 2001: The scientific basis, pp. 92-93.

IPCC (Intergovernmental Panel on Climate Change). 2007. Climate Change 2007: Mitigation. Contribution of Working Group III to the Fourth Assessment Report of the Intergovernmental Panel on Climate Change. en Metz B., Davidson O.R., Bosch P.R., Dave R., Meyer L.A. eds. Cambridge University Press, Cambridge, Reino Unido y Nueva York, 326 p.

Karaosmanoğlu F., Işiğigür A. y Aksoy H.A. Effects of a New Blending Agent on Ethanol-Gasoline Fuels. Energy \& Fuels, volumen 10, 1996:816-820.

Keating E.L. Applied Combustion, 2a ed., CRC Press, Boca Raton, FL, 2007.

Keller J.L. Alcohols as motor fuel? Hydrocarbon Proc., volumen 58, 1979:127-138.

Kwanchareon P., Luengnaruemitchai A. y Jai-In S. Solubility of a Diesel-Biodiesel-Ethanol Blend, its fuel Properties and its Emission Characteristics from Diesel Engine. Fuel, volumen 86, 2006:1053-1061.

Lapuerta M., Armas O. y García-Contreras R. Effect of Ethanol on Blending Stability and Diesel Engine Emissions. Energy \& Fuels, volumen 23, 2009:4343-4354.

Maples R.E. Petroleum Refinery Process Economics, 2a ed., Tulsa: Penn Well Corporation, 2000, 50 p. 
McAloon A., Taylor F. y Yee W. (2000). Determining the Cost of Producing Ethanol from Corn Starch and Lignocellulosic Feedstocks [en linea], Technical Report. Disponible en: http://www.doe. gov/bride.

Mueller S.A., Anerson J.E. y Wallington T.J. A Classroom Demonstration of Water-Induced Phase Separation of AlcoholGasoline Biofuel Blends. J. Chem. Educ., volumen 86, 2009:1045-1048.

Owen K. y Coley T. Automotive Fuels Reference Book, 2a ed., Society of Automotive Engineers, Warrendale, PA, 1995.

PEMEX Refinación. 2008. Especificación No. 107/2008. Hoja técnica de especificaciones.

Pereira R.C.C. y Pasa V.M.D. Effect of Alcohol and Copper Content on the Stability of Automotive Gasolina. Energy \& Fuels, volumen 19, 2005:426-432.

Reyes G. Incremento en los precios del maíz y la tortilla en México. Problemas del Desarrollo, Revista Latinoamericana de Economía, volumen 38, 2007:103-126.

Reyes Y., Aranda D.A.G., Santander L.A.M., Cavado A. y Belchior C.R.P. Action Principle of Cosolvent Additives in EthanolDiesel Blends: Stability Studies. Energy \& Fuels, volumen 23, 2009:2731-2735.

Ribeiro N.M., Pinto A.C., Quintella C.M., Da-Rocha G.O., Teixeira L.S.G., Guarieiro L.L.N., Rangel M.C., Veloso M.C.C., Rezende M.J.C., Serpa da Cruz R., de Oliveira A.M., Torres E.A. y de Andrade J.B. The Role of Additives for Diesel and Diesel Blended (Ethanol or Biodiesel) Fuels: a Review. Energy \& Fuels, volumen 21, 2007:2433-2445.

SAGARPA (Secretaría de Agricultura, Ganadería, Desarrollo Rural, Pesca y Alimentación). 2007, Datos básicos del maíz y series históricas [en línea] Servicio de Información Agroalimentaria y Pesquera. Disponible en:htto://siea.sagarpa.gob.mx. SENER (Secretaría de Energía). Balance Nacional de Energía 2005, Subsecretaria de Planeación y Desarrollo Tecnológico, Sistema de Información Energética, México, 2006.

Torres J. y Molina D. Estudio de la mezcla de gasolina con 10\% de Etanol. Evaluación de propiedades fisicoquímicas. CT \& F Ciencia, volumen 3, 2002:71-82.

Turns S.R. An Introduction to Combustion: Concepts and Applications, $2^{\text {nd }}$ ed., McGraw-Hill, Nueva York, NY, 2000.

Yimin Z., Shiva H., MacLean y Heather L. Environmental and Economic Evaluation of Bioenergy in Ontario, Canada. J. Air $\mathcal{E}$ Waste Manage. Assoc., volumen 57, 2007:919-933.

\section{Este artículo se cita: \\ Citación Chicago}

Castillo-Hernández, Patricia, Alberto Mendoza-Domínguez, Porfirio Caballero-Mata. "Análisis de las propiedades fisicoquímicas de gasolina y diesel mexicanos reformulados con Etanol". Ingeniería Investigación y Tecnología XIII, 03 (2012): 293-306.

\section{Citación ISO 690}

Castillo-Hernández P., Mendoza-Domínguez A., CaballeroMata P. Análisis de las propiedades fisicoquímicas de gasolina y diesel mexicanos reformulados con Etanol. Ingeniería Investigación y Tecnología, volumen XIII (número 3), julio-septiembre 2012: 293-306.

\section{Semblanza de los autores}

Patricia Castillo-Hernández. Obtuvo la licenciatura en ingeniería química en el Instituto Tecnológico de Ciudad Madero y el grado de maestra en ciencias con especialidad en Sistemas Ambientales del Tecnológico de Monterrey (Campus Monterrey).

Alberto Mendoza Domínguez. Es profesor-investigador del Departamento de Ingeniería Química del Tecnológico de Monterrey, Campus Monterrey. Obtuvo su grado de doctor en ingeniería ambiental por el Instituto Tecnológico de Georgia, EU en 2001. Es miembro del Sistema Nacional de Investigadores (SNI) desde el 2002. Sus áreas de investigación están relacionadas principalmente con la ingeniería de la contaminación atmosférica.

Porfirio Caballero-Mata. Es profesor-investigador del Centro de Calidad Ambiental del Tecnológico de Monterrey, Campus Monterrey. Obtuvo su grado de doctor en química por la Universidad Estatal de Louisiana, EU en 1981. Sus áreas de investigación están relacionadas principalmente con la química ambiental. 